\title{
Happy ever after? A new assessment tool for long-term noninvasive ventilation: the $S^{3}$-NIV questionnaire
}

\author{
Anita K. Simonds \\ Affiliation: Royal Brompton and Harefield NHS Foundation Trust, London, UK.
}

Correspondence: Anita K. Simonds, Royal Brompton and Harefield NHS Foundation Trust, Sydney Street, London, SW3 6NP, UK. E-mail: a.simondsarbht.nhs.uk

@ERSpublications

The $\mathrm{S}^{3}$-NIV questionnaire provides a new simple assessment tool for long-term noninvasive ventilation http://ow.ly/BWsY30miiJ6

Cite this article as: Simonds AK. Happy ever after? A new assessment tool for long-term noninvasive ventilation: the $S^{3}$-NIV questionnaire. Eur Respir J 2018; 52: 1801969 [https://doi.org/10.1183/ 13993003.01969-2018].

\begin{abstract}
Over a decade ago, the Eurovent study [1] examined the practice of home mechanical ventilation (HMV) in Europe and showed that the prevalence was increasing and indications for use were growing, but that there were wide inequalities in provision. Services offered varied considerably between countries [2], with no obvious standardisation of care. Subsequent prevalence work worldwide has confirmed the expansion of this area, and randomised trials have established clearer indications for initiation of long-term noninvasive ventilation (NIV), e.g. in chronic obstructive pulmonary disease (COPD) [3-5] and motor neurone disease/amyotrophic lateral sclerosis [6]. Outcomes were originally measured by survival and physiological measures. It is only relatively recently that additional measures important to patients have been addressed. A systematic review of outcomes associated with HMV in disorders other than obstructive lung disease showed that health-related quality of life (HRQoL) was likely to improve with HMV, but these conclusions were based on heterogeneous studies with a variety of HRQoL tools applied [7]. It is broadly accepted that, while generic quality of life assessments such as the 36-item Short-Form Health Survey (SF-36) and Sickness Impact Profile allow comparison across different disorders, they have significant limitations. For example, 10 physical component items in the SF-36 assess ability to perform physical tasks, which may not be possible at baseline in patients with neuromuscular disease. Interestingly, in one trial of HMV in COPD, a disease-specific quality of life score for COPD did not change between control and HMV groups, but several subscales of the SF-36 and a Profile of Mood score deteriorated in the ventilator users [8].
\end{abstract}

The most widely used specific tools for assessing HRQoL in long-term ventilator users (across a range of aetiologies of chronic respiratory failure) are the Severe Respiratory Insufficiency (SRI) Questionnaire, developed by WINDISCH et al. [9], and the Maugeri Respiratory Failure (MRF-26) Questionnaire. A recent comparison of specific questionnaires showed that both SRI and MRF-26 were reliable in patients receiving HMV, but that the SRI covers more psychological health impairments [10].

The SRI comprises seven subscales and covers 49 items, including respiratory complaints, physical functioning, attendant symptoms and sleep, social relationships, anxiety, psychological wellbeing and social functioning [9]. It has been shown to have construct and concurrent validity, can differentiate between 
different diseases (COPD, neuromuscular disease, restrictive chest wall disease and obesity hypoventilation syndrome), has high internal consistency and has been widely translated [11]. However, while suitable for detailed research, the SRI is not easily amenable to monitoring in daily clinical practice. It also does not address ventilator-related side-effects, which may offset health benefits.

In the current issue of the European Respiratory Journal, Dupuis-Lozeron et al. [12] now outline the development and validation of a simpler tool to measure the experience of long-term NIV users: the $S^{3}$-NIV questionnaire. The aim is to provide an easy-to-apply and complete tool to assess respiratory symptoms, sleep and comfort/discomfort with NIV. To derive the tool, items related to respiratory complaints and attendant symptoms and sleep were extracted from the SRI. Important issues to patients associated with comfort and side-effects from NIV were explored initially during qualitative interviews. During exploratory factor analysis the items were further examined, taking into account expert opinion from investigators. In a standard process, reliability was confirmed and construct and discriminant validity explored by comparison of respiratory symptoms with the St George's Respiratory Questionnaire and sleep-related symptoms with Quebec Sleep Questionnaire. Further item analysis and reduction was carried out, leaving 11 questions that patients score on five-point scale from "always true" through to "never true". Interestingly, the cough item became redundant as the statement "There is often mucus in my airways" was more discriminant. The most important questions related to the ventilator were "The mask is uncomfortable", "Air leaks are bothering me" and "Air is too dry". Unexpectedly, the statement "my ventilator is too noisy" was eliminated due to lack of fit with the model. It's probably not a surprise that the perception of symptoms was not correlated with pulmonary function (forced expiratory volume in $1 \mathrm{~s}$ and forced vital capacity) as this is rare in most HRQoL measures. The $S^{3}$-NIV results were also not correlated to adherence, as determined by ventilator downloads. However, it should be noted that the patients on which the $S^{3}$-NIV was generated were experienced long-term users, which is likely to favour better adherence, and this group may have experienced a significant amount of problem-solving throughout the course of their HMV.

Of importance is the nature of the cohort the $\mathrm{S}^{3}$-NIV questionnaire was devised upon. While it comprised $21 \%$ with COPD and $28 \%$ with obesity hypoventilation syndrome, a remarkably high percentage (34\%) had central breathing disturbances during sleep. This case mix may have biased the sleep-related results, as fewer patients with central breathing disorders have raised sleepiness scores. Besides, patients with central sleep disordered breathing do not comprise the largest disease category in many HMV programmes across Europe or worldwide. This group consisted predominantly of elderly and multi-morbid patients with treatment-emergent sleep apnoea, and pure central sleep apnoea due to heart failure, opioids or other causes. Only $12 \%$ of the population evaluated had neuromuscular disease and $5 \%$ had restrictive disorders. It is therefore difficult to be sure whether the results are truly representative of NIV users with neuromuscular and chest wall disease. The authors acknowledge that dependent patients, e.g. those on daytime and nocturnal ventilation, were not included, and it seems unlikely that those with rapidly progressive disorders were represented in significant numbers. The $S^{3}$-NIV questions are also related to mask use, which is an obvious limiting factor for the wider HMV population, as the tool is not designed to assess home tracheostomy-ventilated patients. Evaluation in wider groups of neuromuscular and restrictive chest wall patients is needed, and prospective longitudinal assessment should be carried out to determine how the index responds to change, in order to establish a minimally clinically important difference.

There is no doubt that serial evaluation and patient-reported outcomes are crucial to understanding the full impact of therapies on the lives of NIV users. Furthermore, health commissioners are now routinely seeking this information to make comparative health economic decisions. DupuIs-Lozeron et al. [12] are careful to point out that the $\mathrm{S}^{3}$-NIV is a tool for monitoring and is not intended to be a surrogate measure of general health status or quality of life. The SRI therefore remains a more appropriate tool for randomised controlled trials.

Many of the recent trials have measured health impacts over 12 months or less, but it is not clear whether benefits accrued are retained at 2 years, 3 years, 5 years and more. Does disease progression mean patients reach a later point where burdens of HMV outweigh benefits, despite initial quality of life improvement? Serial, repeated measures are vital here.

As suggested by Dupuis-Lozeron et al. [12], this simplified measure could be incorporated into telemonitoring programmes, so that informed decisions can be made by adapting treatment to a combination of physiological results, ventilator outputs and symptom load. This more comprehensive approach sensibly takes into account patient-related experience. And if it matters to patients, it should matter to us. 
Conflict of interest: A.K. Simonds has nothing to disclose.

\section{References}

1 Lloyd-Owen SJ, Donaldson GC, Ambrosino N, et al. Patterns of home mechanical ventilation use in Europe: results from the Eurovent survey. Eur Respir J 2005; 25: 1025-1031.

2 Farre R, Lloyd-Owen SJ, Ambrosino N, et al. Quality control of equipment in home mechanical ventilation: a European survey. Eur Respir J 2005; 26: 86-94.

3 Köhnlein T, Windisch W, Köhler D, et al. Non-invasive positive pressure ventilation for the treatment of severe stable chronic obstructive pulmonary disease: a prospective, multicentre, randomised, controlled clinical trial. Lancet Respir Med 2014; 2: 698-705.

4 Struik FM, Sprooten RT, Kerstjens HA, et al. Nocturnal non-invasive ventilation in COPD patients with prolonged hypercapnia after ventilatory support for acute respiratory failure: a randomised, controlled, parallel-group study. Thorax 2014; 69: 826-834.

5 Murphy PB, Rehal S, Arbane G, et al. Effect of home noninvasive ventilation with oxygen therapy vs oxygen therapy alone on hospital readmission or death after an acute COPD exacerbation: a randomized clinical trial. JAMA 2017; 317: 2177-2186.

6 Bourke SC, Tomlinson M, Williams TL, et al. Effects of non-invasive ventilation on survival and quality of life in patients with amyotrophic lateral sclerosis: a randomised controlled trial. Lancet Neurol 2006; 5: 140-147.

7 MacIntyre EJ, Asadi L, Mckim DA, et al. Clinical outcomes associated with home mechanical ventilation: a systematic review. Can Respir J 2016; 2016: 6547180.

8 McEvoy RD, Pierce RJ, Hillman D, et al. Nocturnal non-invasive nasal ventilation in stable hypercapnic COPD: a randomised controlled trial. Thorax 2009; 64: 561-566.

9 Windisch W, Freidel K, Schucher B, et al. The Severe Respiratory Insufficiency (SRI) Questionnaire: a specific measure of health-related quality of life in patients receiving home mechanical ventilation. J Clin Epidemiol 2003; 56: 752-759.

10 Oga $\mathrm{T}$, Taniguchi $\mathrm{H}$, Kita $\mathrm{H}$, et al. Comparison of different disease-specific health-related quality of life measurements in patients with long-term noninvasive ventilation. Can Respir J 2017; 2017: 8295079.

11 Ghosh D, Rzehak P, Elliott MW, et al. Validation of the English Severe Respiratory Insufficiency Questionnaire. Eur Respir J 2012; 40: 408-415.

12 Dupuis-Lozeron E, Gex G, Pasquina P, et al. Development and validation of a simple tool for the assessment of home noninvasive ventilation: the $\mathrm{S}^{3}$-NIV questionnaire. Eur Respir J 2018; 52: 1801182. 